\title{
Avaliação dos níveis de aglutininas anti-leptospira em cães no município de Patos - PB, Brasil
}

\section{Evaluation of anti-leptospire agglutinines leves in dogs from Patos - Paraíba state, Brazil}

\author{
Clebert José Alves, ${ }^{\star}$ José Sóstenes Leite de Andrade, ${ }^{\star \star}$ Sílvio Arruda Vasconcellos, ${ }^{\star \star \star}$ Zenaide Macedo de Morais, ${ }^{\star \star \star \star}$ \\ Sérgio Santos de Azevedo, ${ }^{* \star}$ Fabiano Alexandre dos Santos**
}

\begin{abstract}
Resumo
Dentre os animais domésticos urbanos, a principal fonte de infecção da leptospirose humana são os cães, pois estes vivem em contato direto com o homem, podendo eliminar leptospiras vivas através da urina durante meses sem apresentar nenhum sinal clínico. Os cães representam um importante elo de transmissão da leptospirose, visto que aparentemente sadios, podem albergar leptospiras e eliminá-las ao meio ambiente, propício a disseminação para outras espécies, inclusive aos seres humanos. O objetivo do trabalho foi avaliar os níveis de aglutininas anti-leptospira em cães no município de Patos-PB. O material examinado constituiu-se de 114 amostras de soro sangüíneo de cães atendidos no Hospital Veterinário, com ou sem quadro clínico de leptospirose, durante o período de agosto/97 a julho/98. O soro sangüíneo foi processado segundo a técnica de soroaglutinação microscópica (SAM), no Laboratório de Doenças Transmissíveis - CSTR/CAMPUS VII/ UFPB. Após a avaliação dos resultados, observou-se a prevalência de $20 \%$ das amostras com predominância dos seguintes sorotipos: autumnalis, australis, butembo, grippotyphosa, pomona, panamá, wolffi, pyrogenes, icterohaemorrhagiae, hardjo e cynopteri, com destaque para o sorotipo autumnalis $(34,78 \%)$. A análise estatística, utilizando o programa EPIDAT 1.0 , segundo o teste não-paramétrico do qui-quadrado com nível de significância de $5 \%$, possibilitou observar diferenças significativas entre as variáveis estudadas no que diz respeito às regiões administrativas e à natureza dos resultados.
\end{abstract}

Palavras-chave: leptospirose; cães; aglutininas; aglutinação.

\begin{abstract}
Among urban pets, dogs are the principal source of human leptospire infection for they often live in company of man. Dogs tend to eliminate living leptospires through their urine during some months without showing any clinical sign. Those animals represent an important link to leptospire transmission, although apparently in good health condition they may have leptospires and eliminate them in the environment which is favourable to dissemination of other species, even to the man. This research aims to evaluate the anti-leptospire agglutinines levels in dogs from Patos - Paraiba state. In the Veterinary Hospital were collected 114 blood samples of dogs (with or without leptospire predominance) from August/97 to July/98. The blood samples were processed according to the Microscopic Agglutination Technique (MAT) in the Contagious Disease Laboratory - CSTR/ CAMPUS VII/UFPB. After results it was observed the significance of $20 \%$ of the samples with predominance for the following serovars: autumnalis, australis, butembo, grippotyphosa, pomona, panama, wolffi, pyrogenes, icterohaemorrhagiae, hardjo and cynopteri, principally for the serovar autumnalis $(34,78 \%)$. The statistical analysis, using the program EPIDAT 1.0 , according to the test of Qui-square with level of significance of $5 \%$, it was possible to observe important differences among the studied variables concerning administrative region and the nature of the results.
\end{abstract}

Keywords: agglutinines; anti-leptospire; dogs; agglutination.

\section{Introdução}

A leptospirose é uma doença bacteriana que acomete os animais domésticos e o seres humanos, cujo agente etiológico é uma espiroqueta pertencente à Ordem Spirochaetales, Família Leptospiraceae, Gênero Leptospira constituído pelas es- pécies L. interrogans, L. borgpetersenii, L. weilli, L. noguchii, L. santarosai, L. kirchneri e L. feini.

Esta enfermidade vem assumindo grande importância, tanto nos países em desenvolvimento quanto nos desenvolvidos, afetando a saúde animal (Amatredjo et al., 1975 ; Guimarães

*Professor Doutor da Universidade Federal da Paraiba/CSTR/Departamento de Medicina Veterinária, Campus VII, Patos-PB / (Orientador).

**Universidade Federal da Paraíba/CSTR/Departamento de Medicina Veterinária, Campus VII, Patos-PB, Brasil (Bolsista/CNPq).

**Professor Doutor da Universidade Federal da Paraíba/CSTR/Departamento de Medicina Veterinária, Campus VII, Patos-PB / (Orientador).

***Prof. Titular da Faculdade de Medicina Veterinária e Zootecnia (FMVZ) da Universidade de São Paulo (USP), São Paulo-SP, Brasil.

****Laboratório de Zoonoses Bacterianas da Faculdade de Medicina Veterinária e Zootecnia (FMVZ) da Universidade de São Paulo (USP), São Paulo-SP, Brasil. 
et al., 1983 ; Hanson, 1977 ; Michina,1970 ; Tuner, 1967), a economia de produção e a saúde pública, como também a disponibilidade de proteína animal para a população necessitada (Abdusslam, 1975 ; Blenden, 1976 ; Davidson, 1971 ; Faine, 1982 ; Szyfres, 1975).

A ocorrência de leptospirose é variável em diferentes partes do mundo, podendo observar-se tanto de forma esporádica quanto endêmica. Os surtos se reproduzem por exposição à água contaminada com urina ou tecidos provenientes de animais infectados (Tuner, 1967).

Uma vez em contato com a pele lesada ou a mucosa do hospedeiro susceptível, as leptospiras ganham a corrente circulatória e inicia-se a fase de multiplicação do agente no sangue e órgãos afins como: fígado, baço e rins; este é o período de leptospiremia (Faine, 1982 ; Guimarães et al., 1983), que tem duração média de quatro a cinco dias, raramente superando sete dias.

Quando o animal consegue superar a fase aguda, seu sistema imune reage, produzindo anticorpos que antagonizam as leptospiras, presentes nos diversos humores, tecidos e órgãos, fazendo com que elas persistam apenas em algumas áreas do organismo onde os anticorpos ocorrem em níveis baixos, como é o caso da luz dos túbulos renais (Vasconcellos, 1987).

O modelo da evolução da infecção onde a leptospirúria se estabelece após uma fase aguda com sintomatologia evidente caracteriza a modalidade de fonte de infecção referida como "portador convalescente". No entanto, em surtos de leptospirose nos rebanhos de interesse econômico, é comum a existência de indivíduos que apresentam fase aguda assintomática, entrando no período de leptospirúria, inclusive sem demonstrar a presença de anticorpos. Esta última situação apresenta a modalidade de fonte de infecção definida como "portador são", que, pela dificuldade em sua identificação, apresenta ainda maior importância em termos de saúde animal e saúde pública (Vasconcellos, 1987).

Dentre os vários sorotipos que constituem o complexo Leptospira interrogans, são considerados os mais importantes para essa espécie os sorotipos canícola e icterohaemorrhagiae.

O sorotipo canícola determina um quadro clínico de nefrite e gastroenterite, podendo, em alguns casos, a infecção ser assintomática nos cães. Quando ocorre evolução das alterações renais, o animal apresenta uremia, insuficiência renal, enterite hemorrágica, coma e morte em uma alta porcentagem de casos. O sorotipo icterohaemorrhagiae causa nos cães, na maioria das vezes, a síndrome ictérico-hemorrágico, semelhante ao quadro clínico da doença de Weil no homem. Geralmente essa síndrome é aguda e se manifesta clinicamente por hipertermia, prostração, hemorragias em todo o organismo, especialmente nos pulmões e aparelho digestivo, que podem levar à insuficiência hepática (Gírio, 1993 ).

Vários sorotipos foram isolados na espécie canina, encontrando-se nas literaturas citações de $L$. australis, $L$. bataviae, $L$. autumnalis, $L$. hebdomadis e menadencis (Kolochine et al., 1957 ; Brod et al., 1995). Na Argentina, conseguiram isolar o sorotipo pyrogenes de cães (Aguirre, 1969; Brod et al., 1995).

No Brasil e na maioria dos outros países, o isolamento de sorotipos em cães, limita-se a $L$. canícola e $L$. icterohaemorrhagiae (Azevedo, Santos, 1945 ; Brod et al., 1995). No Brasil,observaram-se pela primeira vez cães reagentes para outros sorotipos, $L$. pomona, L. tarassovi, L. hyos, L. sejroe, L.australis, L. bataviae (Castro et al., 1962 ; Brod et al., 1995).

Deste modo, o presente trabalho teve como objetivo pesquisar a presença de aglutininas anti-leptospiras em cães domiciliados no município de Patos.

\section{Material e métodos}

Animais: foram utilizados nesta investigação 114 cães, com ou sem quadro clínico de leptospirose.

Local: os cães foram atendidos na clínica de pequenos animais do Hospital Veterinário da Universidade Federal da Paraíba/ Centro de Saúde e Tecnologia Rural/Campus VII/ Patos-PB.

Coletas: foram realizadas de agosto/97 a julho/98. Por ocasião das coletas, eram anotados dados dos respectivos cães (nome, sexo, idade, raça, proprietário, endereço) objetivando um meIhor acompanhamento destes animais no caso de serem sororeatores. As coletas seguiram metodologia preestabelecida.

As amostras de sangue foram obtidas por punção da veia cefálica com seringas descartáveis, em seguida transferidas para frascos estéreis e enviados ao Laboratório de Doenças Transmissíveis do Centro de Saúde e Tecnologia Rural da Universidade Federal da Paraíba, onde foi realizado o dessoramento em centrífuga a $5 \mathrm{rpm}$ durante 10 minutos, para posterior acondicionamento $\mathrm{a}-20^{\circ} \mathrm{C}$.

As amostras de soro foram submetidas à pesquisa de aglutininas anti-leptospira pelo Laboratório de Zoonoses Bacterianas da Faculdade de Medicina Veterinária e Zootecnia da Universidade de São Paulo pela microtécnica de soroaglutinação microscópica (SAM), que seguiu a metodologia preconizada por Galton et al. (1965), utilizandose uma bateria de antígenos vivos pertencentes ao Laboratório de Zoonoses Bacterianas da Faculdade de Medicina Veterinária e Zootecnia da Universidade de São Paulo, contendo 24 sorovares (L. australis, L. grippotyphosa, L. butembo, L. autumnalis, L. cynopteri, L. icterohaemorrhagiae, L. panamá, L. pomona, L. hardjo, L. canícola, L. wolffi, L. pyrogenes, $L$. bratislava, L.castellonis, L. bataviae, L. whitcombi, $L$. hebdomadis, L. copenhageni, L.javanica, L. shermani, L. tarassovi, $L$. andamana, $L$. patoc e $L$. sentot). Os soros passaram pelas fases de triagem e titulação, com título igual ou superior a 100 e a variante sorológica considerada positiva foi a que apresentou maior título.

Tratamento estatístico: foi realizado utilizando-se o programa EPIDAT 1.0, segundo o teste não-paramétrico do qui-quadrado, com nível de significância de 5\% (Siegel, 1981 ; Vieira et al., 1989).

\section{Resultados}

A Tabela 1 mostra que, das 114 amostras de soro canino examinados, 23 foram reagentes, com título igual ou superior a 100 . Os sorovares, com título igual a 100, foram: $L$. autumnalis, L. grippotyphosa, L. butembo, L. australis; L. icterohaemorrhagiae, L. panamá, L. pomona, L. hardjo, $L$. pyrogenes. Os sorovares com título superior a 100 foram: $L$. autumnalis, L. grippotyphosa, L. butembo, L. australis, L. cynopteri e $L$. wolffi. A análise estatística utilizando o programa EPIDAT 1.0; através do teste do qui-quadrado não revelou diferenças significativas entre as variáveis estudadas. 
A Tabela 2 mostra os resultados dos exames de soros sangüíneos de cães examinados através da microtécnica aplicada à leptospirose, segundo o número de amostras e o sorotipo reator. Observou-se nesta tabela que os sorotipos mais freqüentes foram: $L$. autumnalis (34,78\%), L. grippotyphosa $(13,04 \%)$, L. butembo $(13,04 \%)$, L. australis $(8,70 \%)$.

A Tabela 3 apresenta os resultados dos exames de soro sangüíneo em cães através da microtécnica, segundo o sexo e a natureza dos resultados. Observou-se nesta tabela que, dos 79 soros de cães machos, $18(15,8 \%)$ reagiram para um ou mais sorotipo, enquanto que dos 35 soros de fêmeas apenas $5(4,4 \%)$ reagiram também para um ou mais sorotipo. $A$ análise estatística pelo programa do EPIDAT 1.0 não mostrou diferenças significativas entre as variáveis estudadas.

A Tabela 4 mostra os resultados dos exames de soros sangüíneos de cães através da microtécnica aplicada à leptospirose, segundo a faixa etária e a natureza dos resultados. Observou-se pela tabela que: dos 28 soros de cães com idade inferior ou igual a 1 ano, apenas $2(1,9 \%)$ foram sororeatores. Das 77 amostras de soro de cães com idade superior a 1 ano, $18(17,1 \%)$ foram soro-reatores. Não se observaram diferenças significativas entre as variáveis, utilizando-se o programa EPIDAT 1.0 para as análises estatísticas.

A Tabela 5 apresenta os resultados de exames de soro sanguíneo de cães através da microtécnica aplicada à leptospirose, segundo a região administrativa e a natureza dos resultados. Dos 114 soros coletados, obtiveram-se 23 soros reatores distribuídos da seguinte forma: 17 na Regional I, 3 na Regional II, 1 na Regional III e 2 na Regional IV. A análise estatística utilizando o programa EPIDAT 1.0, através do teste do qui-quadrado, não revelou diferenças significativas entre as variáveis estudadas.

Tabela 1: Resultados dos exames de amostras de soro canino através da microtécnica aplicada à leptospirose, segundo os sorotipos prevalentes e o título. Patos-PB, agosto/ 97 a julho/98

\begin{tabular}{l|ccc}
\hline \multirow{2}{*}{ Sorotipos } & \multicolumn{3}{|c}{ Título } \\
\cline { 2 - 4 } Australis & 1 & 1 & 2 \\
Autumnalis & 2 & 6 & 8 \\
Butembo & 1 & 2 & 3 \\
Cynopteri & - & 1 & 1 \\
Grippotyphosa & 1 & 2 & 3 \\
Hardjo & 1 & - & 1 \\
Icterohaemorrhagiae & 1 & - & 1 \\
Panama & 1 & - & 1 \\
Pomona & 1 & - & 1 \\
Pyrogenes & 1 & - & 1 \\
Wolffi & - & 1 & 1 \\
Total & 10 & 13 & 23 \\
\hline
\end{tabular}

Tabela 2: Resultados de exames de amostras de soro canino através da microtécnica aplicada à leptospirose, segundo o número de amostras e o sorotipo reator. Patos-PB, agosto / 97 a julho/98

\begin{tabular}{|c|c|c|}
\hline Sorotipo reator & Amostras reatoras & Total (\%) \\
\hline andamana & $\cdots$ & $\cdots$ \\
\hline australis & 02 & 8,70 \\
\hline autumnalis & 08 & 34,78 \\
\hline bataviae & -- & --- \\
\hline bratislava & -- & $\cdots$ \\
\hline butembo & 03 & 13,04 \\
\hline canicola & $--\cdot$ & $--\cdot$ \\
\hline castellonis & --- & -.- \\
\hline copenhageni & $-\cdot$ & --- \\
\hline cynopteri & 01 & 4,35 \\
\hline grippotyphosa & 03 & 13,04 \\
\hline hardjo & 01 & 4,35 \\
\hline hebdomadis & -- & -.. \\
\hline icterohaemorrhagiae & 01 & 4,35 \\
\hline javanica & $\cdots$ & -- \\
\hline panamá & 01 & 4,35 \\
\hline patoc & $--\cdot$ & $\cdots$ \\
\hline pomona & 01 & 4,35 \\
\hline pyrogenes & 01 & 4,35 \\
\hline sentot & $\cdots$ & --- \\
\hline shermanii & --- & -- \\
\hline tarassovi & $--\cdot$ & $\cdots$ \\
\hline whitcombi & --- & $\cdots$ \\
\hline wolffi & 01 & 4,35 \\
\hline Total & 23 & 100,00 \\
\hline
\end{tabular}

Tabela 3: Resultados dos exames de amostras de soros caninos através da microtécnica aplicada à leptospirose, segundo o sexo e a natureza do resultado. Patos-PB, agosto/97 a julho/98

\begin{tabular}{c|ccc}
\hline \multirow{2}{*}{ Reator } & \multicolumn{3}{|c}{ Sexo } \\
\cline { 2 - 4 } & Machos & Fêmeas & Total \\
\hline Sim & $18(15,8 \%)$ & $5(4,4 \%)$ & $23(20,2 \%)$ \\
Não & $61(53,5 \%)$ & $30(26,3 \%)$ & $91(79,8 \%)$ \\
Total & $79(69,3 \%)$ & $35(30,7 \%)$ & $114(100 \%)$ \\
\hline
\end{tabular}

Qui-quadrado observado: 1, 09

Qui-quadrado crítico ao nivel de $5 \%: 3,84$ 
Tabela 4: Resultados de exames de amostras de soro canino através da microtécnica aplicada à leptospirose, segundo a faixa etária e a natureza dos resultados. Patos-PB, agosto/97 a julho/98

\begin{tabular}{l|ccr}
\hline \multirow{2}{*}{ Reator } & \multicolumn{3}{|c}{ Idade } \\
\cline { 2 - 4 } & $\begin{array}{c}\text { Inferior ou Igual } \\
\text { a 1 ano(\%) }\end{array}$ & $\begin{array}{c}\text { Superior a 1 ano } \\
(\%)\end{array}$ & Total* $^{\star}$ \\
\hline Sim & $02(1,9 \%)$ & $18(17,1 \%)$ & $20(19 \%)$ \\
Não & $26(24,8 \%)$ & $59(56,2 \%)$ & $85(81 \%)$ \\
Total & $28(26,7 \%)$ & $77(73,3 \%)$ & $105(100 \%)$ \\
\hline
\end{tabular}

Qui-quadrado observado: 3, 52

Qui-quadrado crítico ao nível de $5 \%: 3,84$

* Idade Desconhecida (9 cães)

Tabela 5: Resultados dos exames de amostras de soro canino através da microtécnica aplicada à leptospirose, segundo a região administrativa e a natureza dos resultados Patos$\mathrm{PB}$, agosto/97 a julho/98.

\begin{tabular}{c|ccccc}
\hline \multirow{2}{*}{ Reator } & \multicolumn{5}{|c}{ Região } \\
\cline { 2 - 6 } & $\begin{array}{c}\text { Regional } \\
\text { I }\end{array}$ & $\begin{array}{c}\text { Regional } \\
\text { II }\end{array}$ & $\begin{array}{c}\text { Regional } \\
\text { III }\end{array}$ & Regional IV & Total \\
\hline Sim & 17 & 03 & 01 & 02 & 23 \\
Não & 55 & 10 & 11 & 15 & 91 \\
Total & 72 & 13 & 12 & 17 & 114 \\
\hline
\end{tabular}

Qui-quadrado observado : 2,36

Qui-quadrado crítico ao nivel de $5 \%: 7,81$

Regional I - Jatobá, Mutirão, Monte Castelo, Alto da Tubiba.

Regional II - Liberdade, Santo Antônio, Jardim Guanabara, Bivar Olinto.

Regional III - Belo Horizonte, Jardim Queiróz, Jardim Europa.

Regional IV - Brasília, Califórnia, Salgadinho, Centro, Vila Cavalcante.

\section{Discussão}

Os dados obtidos nesta pesquisa em relação à prevalência de animais reatores foi de $20 \%$, o que vem confirmar as variações nas freqüências anteriormente citadas por Castro et al. (1962), com 14,1\%; Herrer (1967), com 16\%; SILVA et al. (1973), com $84,5 \%$.

\section{Referências bibliográficas}

ABDUSSLAM. M. Situación mundial del problema de la leptospirose. ORGANIZAÇÃO MUNDIAL DE SAÚDE. REUNIÓN INTERAMERICANA SOBRE EL CONTROL DE LA FIEBRE AFTOSA Y OTRAS ZOONOSIS. 8., Guatemala, p. 142-153. (Publicación Científica, 316). 1975

AGUIRRE, W.G., SILVA, I. Isolamento in Argentina di um ceppo di leptospira apartenente al serotipo pyrogenis. Ann Inst Super Sanitá, v. 5, p. 1956. 1969.

AMATREDJO, A. \& CAMBELL, R.S.F. Bovine leptospirosis. Vetrinary Bulletin. n. 45, p. 875-890, 1975.
Embora resultados de pesquisas semelhantes tenham apresentado diferenças, talvez isto possa ser explicado, de alguma forma, pela variedade de fatores que influenciam na ocorrência da leptospirose com destaque para topografia, região, temperatura, umidade, precipitações pluviométricas, reservatórios selvagens (preás, raposas, gambás etc.), reservatórios domésticos e outros fatores ambientais.

No presente trabalho, verificou-se a maior prevalência de aglutininas anti-leptospira dos respectivos sorotipos $L$. autumnalis, L. grippotyphosa, L. butembo, L. australis, diferentemente dos achados clássicos, onde prevalecem os sorotipos canícola e icterohaemorrhagiae. A presença destes sorotipos encontrados, bem como a de outros citados por Rodrigues et al. (1997), causa preocupação que vai desde a contaminação do ambiente até a proteção específica, visto que não existe imunidade cruzada, pois no mercado existem as vacinas compostas, basicamente, pelos sorotipos canícola e icterohaemorrhagiae.

A interpretação de títulos baixos nas reações positivas exige muito cuidado, pois segundo Farina, 1968, títulos de 100 ou 200 podem aparecer no ínicio da doença bem como tardiamente, em alguns casos. Por outro lado, Santa Rosa (1970) salienta que título 100 é suficiente para confirmar o diagnóstico. Wolh (1996) considera que um animal apresentando um título superior a 800 associado ao histórico do paciente com evidências laboratoriais e clínicas compatíveis já seria suficiente para fechar o diagnóstico para leptospirose. Em nossas pesquisas, verificou-se a predominância dos títulos superiores a 100. Estes títulos variaram entre 100 e 800 , indicando desta forma que a conduta em relação à interpretação dos resultados sorológicos deve merecer toda a atenção, seja do solicitante particular, seja do pesquisador, quando das suas análises.

A avaliação da Tabela 5 permitiu concluir que os maiores índices de animais reatores foram provenientes dos bairros da periferia do município de Patos, fato este que poderia estar associado, particularmente, aos problemas de infra-estrutura como coleta irregular de lixo, problemas com saneamento básico, baixo nível de instrução, presença de cães vadios e sem controle.

\section{Conclusão}

A avaliação dos dados obtidos permite concluir que a leptospirose existe no município de Patos-PB, sendo necessário o estabelecimento de medidas saneadoras para o controle desta enfermidade.

AZEVEDO, A. G. \& SANTOS, J.S. Sobre a ocorrência de leptospirose no Rio de Janeiro. CONGRESSO BRASILEIRO DE MEDICINA VETERINÁRIA, 3. ed. Porto Alegre. Anais... 1945, p. 115-163.

BLENDEN, D.C. Aspectos epidemiológicos de la leptospirosis. ORGANIZAÇÃOMUNDIAL DE SAÚDE. REUNIÓNINTERAMERICANASOBRE EL CONTROL DE LA FIEBRE AFTOSA Y OTRAS ZOONOSIS. Guatemala, 8., 1975. Washington, D.C., Organización Panamericana de la Salud, p. 160-168. (Publicación Científica, 316). 1976.

BROD, C. S., ROSADO, R.L.I., TEIXEIRA, M.M., ÁVILA, M.M., FURTADO, L.R.I., MARTINS, L.F.S. Aglutininas anti-leptospiras em cães na área de influência do C.C.Z, Pelotas, noano de 1995. CONGRESSOBRASILEIRODEMEDICINA VETERINÁRIA, 24., Goiânia, Anais...1996, 251p. 
CASTRO, A. F.P., SANTA ROSA, C. A., TROISE, C. et al. Leptospirose canina em São Paulo: inquérito sorológico e isolamento da leptospira ictero. Arq Inst Biol, São Paulo, v. 23, p.199-205. 1962.

DAVIDSON, K.R. Leptospira hardjo infeccion in man associated with an outbreak in a dairy herd. Australian Veterinary Journal. v. 47, 408 p. 1971.

FAINE; S. Guidelines for the control of leptospirosis. Geneva, World Health Organization, 171 p. (Who off set publicacion, 67). 1982.

FARINA, R. Contributo alla conoscenza delle leptospirosi del cane. Zooprofilassi, n. 13, p. 7-11, 1968.

GALTON,M.M., SULZER,C.R, SANTA ROSA, C.A., FIELDS, M.J. Aplication of a Microtecnhique to the Aglutination Test for Leptospiral Antibodies. Applied Microbiology, v. 13, p. 81-85.1965.

GíRIO, R.J.S. Abordagem clínica da leptospirose animal. ENCONTRO NACIONAL EM LEPTOSPIROSE. 3., Rio de Janeiro, Anais... 1993, $157 \mathrm{p}$.

GUIMARÃES, M.C., CÔRTES, J.A., VASCONCELLOS, S.A., ITO, F.H. Epidemiologia e controle de leptospirose em bovinos. $\mathrm{Pa}-$ pel do portador e seu controle terapêutico. COMUNICAÇÕES CIENTIIFICAS DA FACULDADE DE MEDICINA VETERINÁRIA E ZOOTECNIA DA UNIVERSIDADE DE SÃO PAULO, v. 6/7, p. 21 $34,1982 / 1983$.

HANSON, L.E. Imunology of bacterial diseases, with special reference to leptospirosis. Journal of the American Veterinary Medical Association. v. 170, p. 991-994, 1977.

HERRER, A., LECERAS, J., MENDES. O. Identificación de las cepas de leptospiras presentes en el perro y el gato y incidência de la infección. Div. Est. Epid. e Inv. Espec. del Inst. Nac. Salud Pub, Lima, Peru - Rev. Med Exp, 120 p. 1967.

KOLOCHINE, E., GORET, P., BAUCHET, A. Enquete serologique sur la frequence en France des infections leptospirosiques canines. Bull Acad Vet Fr, v. 30, p. 423-426, 1957.
MICHINA, S. W. Leptospirosis. Veterinary Record, v. 86, p. 484-496, 1970. RODRIGUES, C.G., VIEIRA, A. M., PRETTO, L.G., METTIFOGO, E., MULLER, E.E., FREITAS, J.C. Sorotipos detectados através da prova de aglutinação microscópica em cães com suspeita clínica de leptospirose. CONGRESSOBRASILEIRO DEMEDICINA VETERINÁRIA (COMBRAVET). 25., Gramado, 1997, p. 306. 1997.

SANTA ROSA, C. A., CASTRO, A.F., SILVA, A. S. et al. Nove anos de leptospirose no Instituto Biológico de São Paulo. Rev Inst Adolfo Lutz, v. 29/30, p. 19-27, 1969/1970.

SANTA ROSA, C. A. Diagnóstico laboratorial das leptospiroses. Revista de Microbiologia, v. 1, p. 97-109, 1970.

SILVA, A. S.; TERUYA, M. J; SANTA ROSA, C. A. et al. Pesquisa de aglutininas anti-leptospira em soros de cães clinicamente suspeitos, na cidade de São Paulo; Arq Inst Biol, São Paulo, p. 336-340, 1973.

SIEGEL, S. Estatística não-paramétrica para as ciências do comportamento. São Paulo: MC Graw Hill, 350 p. 1981.

SZYFRES, B. La leptospirosis como problema de salud humana y animal en América Latina y el area del Caribe. REUNIÓN INTERAMERICANA SOBRE EL CONTROL DE LA FIEBRE AFTOSA Y OTRAS ZOONOSIS. 8., Guatemala, p. 125-141. (Publicación Científica, 316). 1975.

TUNER, L.H. leptospirosis I. Transactions of the Royal Society of Tropical Medicine and Hygiene, n. 61, p. 842-855, 1967.

VASCONCELLOS, S. A. O papel dos reservatórios na manutenção de leptospirose na natureza. Comunicações Científicas da Faculdade de Medicina Veterinária e Zootecnia da Universidade de São Paulo, n. 11, p. 17-24, 1987.

VIEIRA, S., HOFFMANN, R. Estatística experimental. São Paulo : Atlas, 179 p. 1989.

WOHL, J. S. Canine Leptospirosis. The Compendium, n. 11, v. 18, 1996.

\begin{tabular}{|c|} 
HEXÁGONO QUÍMICA E EQUIPAMENTOS \\
PARA LABORATÓRIOS LTDA. \\
( Distribuidor MERCK, VWR, WTW, BRAND ) \\
\\
RUADAS OFICINAS, 167-ENGENHO DE DENTRO - CEP 20770-010 \\
FAX: (21) 595-6055 - PABX: (21) 597-1231
\end{tabular}

